\title{
SELF-SIMILAR SOLUTIONS FOR THE 2-D BURGERS SYSTEM IN INFINITE SUBSONIC CHANNELS
}

\author{
KYUNGWOO SONG
}

\begin{abstract}
We establish the existence of weak solutions in an infinite subsonic channel in the self-similar plane to the two-dimensional Burgers system. We consider a boundary value problem in a fixed domain such that a part of the domain is degenerate, and the system becomes a second order elliptic equation in the channel. The problem is motivated by the study of the weak shock reflection problem and 2-D Riemann problems. The two-dimensional Burgers system is obtained through an asymptotic reduction of the 2-D full Euler equations to study weak shock reflection by a ramp.
\end{abstract}

\section{Introduction}

In this paper, we are considering self-similar solutions of the two-dimensional Burgers system

$$
\left\{\begin{array}{r}
u_{t}+u u_{x}+v_{y}=0 \\
v_{x}-u_{y}=0
\end{array}\right.
$$

where $u, v$ are the velocity components in the 2-D full Euler equations. Brio and Hunter [1] obtained the system through an asymptotic reduction of the 2-D full Euler equations to study weak shock reflection by a ramp. C. S. Morawetz [6] also established the system to model shock reflection by asymptotic reduction from the transonic full potential equation.

To simplify the oblique-shock-reflection problem, it is assumed generally the wedge angle is small, or the incident shock is weak. It is known that various conditions on the angle and the shock strength yield regular or irregular reflections. When, in particular, the angle is in the order of the square-root of the shock strength, the system arises to describe the solution at the core of wave-wall interaction. So it is expected that (1.1) contains the information on

Received August 7, 2008.

2000 Mathematics Subject Classification. 35J70, 35M10, 35L65.

Key words and phrases. changing-type equations, degenerating quasilinear elliptic equations, self-similar solutions, 2-D full Euler equations.

This work was supported by the Korea Research Foundation Grant funded by the Korean Government (MOEHRD, Basic Research Promotion Fund) (KRF-2008-331-C00028). 
transition of regular reflection to irregular reflection which includes the various Mach reflections and von Neumann wave, etc. More details are in [10]. The 2D Burgers system also arises in nonlinear acoustics and nonlinear geometrical optics [8]. This system of equations is also called unsteady transonic small disturbance equations.

In weak regular reflection, the state behind the reflected shock is supersonic and constant. The flow becomes subsonic further downstream and the system of equations in self-similar coordinates degenerates at the sonic line. The reflected shock becomes transonic and begins to curve there. Since the position of the shock is unknown, the shock reflection problem reduces a free boundary problem. Several results have been obtained on degenerating elliptic equations for the system. Refer to $[2,3]$ for free boundary problems of transonic small disturbance equations on bounded domains. In [4], a $C^{2}$-solution of a system of 2-D Burgers equations on a fixed bounded domain was obtained through quasilinear degenerate elliptic problems.

In self-similar coordinates $(\xi, \eta)=(x / t, y / t)$ the system becomes

$$
\left\{\begin{aligned}
-\xi u_{\xi}-\eta u_{\eta}+\left(u^{2} / 2\right)_{\xi}+v_{\eta} & =0, \\
-v_{\xi}+u_{\eta} & =0 .
\end{aligned}\right.
$$

Introducing the potential function $\varphi$ such that $\varphi_{\xi}=u, \varphi_{\eta}=v$, we can rewrite (1.2) in the form

$$
\left(\varphi_{\xi}-\xi\right) \varphi_{\xi \xi}-\eta \varphi_{\xi \eta}+\varphi_{\eta \eta}=0 .
$$

This is a second order quasilinear equation of mixed type and the coefficients of the second order terms depend on the first order derivatives. We can also derive an equation for $u$ directly from (1.2) in the form

$$
(u-\xi) u_{\xi \xi}-\eta u_{\xi \eta}+u_{\eta \eta}+\left(u_{\xi}-1\right) u_{\xi}=0
$$

whose coefficients of the second order terms depend on $(u, \xi, \eta)$ which is less nonlinear. We also note both forms (1.2) and (1.4) have the same characteristics

$$
\frac{d \xi}{d \eta}=-\frac{\eta}{2} \pm \sqrt{\frac{\eta^{2}}{4}+\xi-u}
$$

which change types across the parabolic line $u=\xi+\eta^{2} / 4$. In another coordinate system $(\rho, \eta)$ with $\rho=\xi+\eta^{2} / 4$, the 2 -D Burgers system becomes

$$
\begin{aligned}
(u-\rho) u_{\rho}-\frac{\eta}{2} u_{\eta}+v_{\eta} & =0, \\
\frac{\eta}{2} u_{\rho}-v_{\rho}+u_{\eta} & =0 .
\end{aligned}
$$

We note that for a given solution $u(\xi, \eta)$, the hyperbolic region is $\frac{\eta^{2}}{4}+\xi-u=$ $\rho-u>0$ on which the flow become supersonic and the elliptic region is $\frac{\eta^{2}}{4}+\xi-u=\rho-u<0$ on which the flow become subsonic. That is, for a given constant state $\left(u_{0}, v_{0}\right)$, the system is hyperbolic outside $\rho=\xi+\frac{\eta^{2}}{4}=$ 
$u_{0}$ and elliptic inside. So the parabola is a degenerating boundary and the unbounded elliptic region is obtained. Eliminating the variable $v$ from two previous equations and introducing new variables $x=\rho, y=\eta$ to make the degenerate boundary straight, we have a second order differential equation for $u$ which is a mixed-type equation:

$$
(u-x) u_{x x}+u_{y y}+\left(u_{x}-\frac{1}{2}\right) u_{x}=0
$$

or

$$
\left((u-x) u_{x}\right)_{x}+u_{y y}+\frac{1}{2} u_{x}=0 .
$$

In the paper, we are interested in subsonic solutions of the 2-D Burgers system. We believe that it is helpful to fully understand the subsonic regions locally before we solve the 2-D Burgers system in the whole plane. Thus it is necessary to investigate the property of solutions in unbounded elliptic regions and then a global solution to the 2-D Burgers system can be patched together by pieces along characteristic lines, sonic curves, shock waves or other boundaries. Hence it is meaningful to consider the problem in an infinite channel which will be turned out to be an elliptic region. Let

$$
\Omega=\{-\infty<x<0,-1<y<1\} \subset \mathbb{R}^{2}
$$

be an infinite channel.

In this paper, we find a weak solution $u=u(x, y)$ to the problem

$$
\left\{\begin{aligned}
(u-x) u_{x x}+u_{y y}+\left(u_{x}-\frac{1}{2}\right) u_{x} & =0, \\
\left.u\right|_{\partial \Omega} & =g,
\end{aligned}\right.
$$

where the function $g \in C^{0, \beta}(\partial \Omega)(0<\beta<1)$ satisfies $g>x$ on $\partial \Omega \backslash\{x=0\}$, $g=0$ on $\{x=0\} \subset \partial \Omega$, and $g \rightarrow-N$ as $x \rightarrow-\infty$ for some constant $N>0$.

Our approach is based on the weak solution approach close to those of Y. Zheng [9] and K. Song [7] applied to the pressure-gradient system.

Theorem 1.1. Let $\Omega$ be an infinite channel in $\mathbb{R}^{2}$. Then there exists a weak solution $u$ to (1.7) with $u \in H_{\text {loc }}^{1}(\Omega)$. The solution takes its boundary value in the sense $(u-g)^{3 / 2} \chi \in H_{0}^{1}(\Omega)$ for a localizer $\chi$ of the boundary, where $g$ is a bounded smooth extension to $\Omega$. In addition, $u-x>0$ in the whole domain.

\section{Main results}

Let us regard the domain $\Omega$ as $\Omega=\bigcup_{j=1}^{\infty} \Omega_{j}$ where

$$
\Omega_{j}=\Omega \cap\left\{(x, y) \in \mathbb{R}^{2} \mid-j<x<0\right\}
$$

is a bounded subdomain with $\Omega_{j} \nearrow \Omega$. We extend the boundary data $g$ to the whole domain such that $g \in C^{2}(\Omega) \cap C^{0, \beta}(\bar{\Omega})$ satisfies $g>x$ and

$$
\max _{0 \leq|\alpha| \leq 2} \sup _{\Omega}\left|D^{\alpha} g\right|<\infty \text {. }
$$


Then we consider a Dirichlet problem of (1.5) in each bounded subdomain $\Omega_{j}$ with a boundary condition $\left.g\right|_{\partial \Omega_{j}}$.

Before we proceed, we cite some useful results from [4]. In [4], the existence and regularity of solutions of the 2-D Burgers system in a bounded domain $U$ with a proper boundary condition $\left.h\right|_{\partial U}$ has been considered. Since the original equation (1.5) is quasilinear, they consider a modified regularized equation

$$
\left((A(x, y, u)+\varepsilon) u_{x}\right)_{x}+u_{y y}+\frac{1}{2} u_{x}=0
$$

in order to get an elliptic equation, where

$$
A(x, y, z)=\left\{\begin{array}{cl}
z-x & : \quad z-x \geq 0 \\
0 & : \quad z-x<0
\end{array}\right.
$$

It is shown that there exists a solution $u^{\varepsilon} \in C^{2, \alpha}(U) \cap C^{0, \alpha}(\bar{U})$ of $(2.1)$ in a bounded domain $U$ with a uniform exterior cone condition on $\partial U$ for some $0<\alpha<1$ and $h \in W^{1,2}(U) \cap C^{0, \beta}(\bar{U})$ (Theorem 2.1 in [4]). We point out this result is still valid for the data $h \in C^{2}(U) \cap C^{0, \beta}(\bar{U})$ with $\left|D^{2} h\right|<\infty$. According to Lemma 2.4 in [4], the solution $u^{\varepsilon}$ satisfies $u^{\varepsilon}-x>0$ on $U$. Finally, they have showed the existence of the limit solution

$$
u \in C^{2}(U) \cap C^{0}(\bar{U})
$$

satisfying $u>h$ (Theorem 4.2 in [4]). On the other hand, the equation (2.1) becomes

$$
\left((u-x+\varepsilon) u_{x}\right)_{x}+u_{y y}+\frac{1}{2} u_{x}=0 .
$$

Now in order to get the maximum principle we regard (2.3) as a linear equation and then apply the Schauder fixed point theory as in [4]. Then the limit solution $u$ in $(2.2)$ satisfies

$$
|u| \leq \max _{\partial U}|h| \text { in } U .
$$

Now let us go back to the 2-D Burgers system in each bounded subdomain $\Omega_{j}$ and each solution $u^{j} \in C^{2}\left(\Omega_{j}\right) \cap C^{0}\left(\bar{\Omega}_{j}\right)$ in $\Omega_{j}$. Let us extend the solution $u^{j}$ obtained into the whole domain:

$$
u^{j}=\left\{\begin{array}{rll}
u^{j} & : & \Omega_{j} \\
g & : & \Omega \backslash \Omega_{j} .
\end{array}\right.
$$

By the maximum principle and the property of $g$, there exists some number $L$ independent of $j$ such that $\left|u^{j}\right|<L$ in the whole domain $\Omega$.

In order to construct solutions of (1.5) in the whole domain we consider a sequence of $\left\{u^{j}\right\}$. By the method of Lemma 2.4 in [4] (or Lemma 2.3 in [7]) we can show easily the uniform ellipticity condition. Here let us give its proof for concreteness. 
Lemma 2.1 (Lemma 2.4 in [4]). For every nonnegative function $\phi \in C_{0}^{3}\left(\overline{\Omega_{*}}\right)$ which is strictly positive in the interior of $\Omega_{*} \in\left\{\Omega_{j}\right\}$ there exists a $\delta>0$ independent of $j$ such that

$$
u^{j}-x>\delta \phi
$$

on a subdomain $\Omega_{*}$ for sufficiently large $j$.

Proof. Assume for a given function $\phi$, it is not possible to find a $\delta>0$ such that $u^{j}-x-\delta \phi>0$ in $\Omega_{*}$ for all sufficiently large $j$. Then for each $\delta>0$, there exists a point $p=\left(x_{0}, y_{0}\right) \in \Omega_{*}$ such that $u^{j}(p)-x_{0}-\delta \phi(p) \leq 0$. That is, $p$ is a local minimum point. Then at the point

$$
\begin{aligned}
& \nabla\left(u^{j}-x-\delta \phi\right)=0, \\
& \left(u^{j}-x\right)\left(u^{j}-x-\delta \phi\right)_{x x}+\left(u^{j}-x-\delta \phi\right)_{y y} \geq 0 .
\end{aligned}
$$

The equation (1.5) becomes

$$
\begin{gathered}
\left(u^{j}-x\right)\left(u^{j}-x-\delta \phi\right)_{x x}+\left(u^{j}-x-\delta \phi\right)_{y y}+\left(u^{j}-x\right)(x+\delta \phi)_{x x} \\
+(x+\delta \phi)_{y y}+\left(\delta \phi_{x}+\frac{1}{2}\right)\left(\delta \phi_{x}+1\right)=0
\end{gathered}
$$

We note that the left hand side of (2.5) is strictly positive at the point $p$ if we take small enough $\delta>0$ such that

on $\Omega_{*}$. Thus we are led to a contradiction.

$$
\frac{1}{2}+\delta\left(\left(u^{j}-x\right) \phi_{x x}+\phi_{y y}+\delta \phi_{x}^{2}+\frac{3}{2} \phi_{x}\right)>0
$$
have

$$
\begin{aligned}
\left(\psi^{j}+g-x\right)\left(\psi^{j}+g\right)_{x x} & +\left(\psi^{j}+g\right)_{y y} \\
& +\left(\left(\psi^{j}+g\right)_{x}-\frac{1}{2}\right)\left(\psi^{j}+g\right)_{x}=0 .
\end{aligned}
$$

Let us show the existence of a subsequence $\left\{\psi^{j_{k}}\right\}$ of the sequence $\left\{\psi^{j}\right\}$ and its limit function $\psi \in H_{l o c}^{1}(\Omega)$.

Lemma 2.2. There exist a subsequence $\left\{\psi^{j_{k}}\right\}$ of the sequence $\left\{\psi^{j}\right\}$ and a function $\psi \in H_{l o c}^{1}(\Omega)$ such that

$$
\begin{gathered}
\psi^{j_{k}} \rightarrow \psi \quad \text { in } \quad L_{l o c}^{2}(\Omega), \\
\nabla \psi^{j_{k}} \rightarrow \nabla \psi \quad \text { in } \quad L_{l o c}^{2}(\Omega) .
\end{gathered}
$$

Proof. Let $\Omega_{j}$ be a subdomain and $\Omega_{R} \in\left\{\Omega_{j}\right\}$ with a compact $K \subset \subset \Omega_{R} \subset \Omega_{j}$. Define a non-negative function $\zeta \in C^{\infty}(\Omega)$ with

$$
\zeta: \Omega \rightarrow[0,1] \text { such that }\left.\zeta\right|_{K}=1 \text { and } \operatorname{supp} \zeta:=K_{0} \subset \subset \Omega_{R} \text {. }
$$

For convenience, let $\psi^{j}:=\psi$. Multiply the equation (2.6) by $\psi \zeta^{2}$ to find

$$
\zeta^{2} \psi(\psi+g-x)(\psi+g)_{x x}+\zeta^{2} \psi(\psi+g)_{y y}+\zeta^{2} \psi\left((\psi+g)_{x}-\frac{1}{2}\right)(\psi+g)_{x}=0
$$


Integration over $\Omega_{R}$ makes

$$
\begin{aligned}
& \quad \int \zeta^{2}\left(\psi_{x}^{2}(\psi+g-x)+\psi_{y}^{2}\right) \\
& =\int\left\{\zeta^{2} \psi(\psi+g-x) g_{x x}-2 \zeta \zeta_{x} \psi(\psi+g-x) \psi_{x}\right. \\
& \left.\quad+\zeta^{2} \psi \psi_{x} g_{x}+\frac{1}{2} \zeta^{2} \psi \psi_{x}+\zeta^{2} g_{y y} \psi-2 \zeta \zeta_{y} \psi \psi_{y}+\zeta^{2} g_{x}^{2} \psi-\frac{1}{2} \zeta^{2} \psi g_{x}\right\} .
\end{aligned}
$$

If we use the Cauchy inequality with $\varepsilon$, the above equation becomes

$$
\int_{K_{0}} \zeta^{2}\left((\psi+g-x) \psi_{x}^{2}+\psi_{y}^{2}\right) \leq C+C \varepsilon \int_{K_{0}} \zeta^{2} \psi_{x}^{2},
$$

where $C$ is a constant independent of $j$. By $(2.4), m:=\min _{K}(\delta \phi)>0$. If we take $\varepsilon>0$ so that $m-C \varepsilon=\frac{m}{2}$, then $\int_{K}\left(\frac{m}{2} \psi_{x}^{2}+\psi_{y}^{2}\right) d x d y \leq C$ which yields that $\int_{K}\left|\nabla \psi^{j}\right|^{2} d x d y \leq C$, where $C$ is a constant depending on $\Omega_{R}$, independent of $j$ and $\Omega_{j}$. By standard results we get that $\left\{\psi^{j}\right\}$ is bounded in $H^{1}\left(\Omega_{R}\right)$ and by diagonal extraction it follows that there exist a subsequence $\left\{\psi^{j_{k}}\right\}$ of the sequence $\left\{\psi^{j}\right\}$ and a function $\psi \in H_{l o c}^{1}(\Omega)$ such that

$$
\psi^{j_{k}} \rightarrow \psi \quad \text { in } \quad L_{l o c}^{2}(\Omega), \quad \text { and } \quad \nabla \psi^{j_{k}} \rightarrow \nabla \psi \text { in } \quad L_{l o c}^{2}(\Omega) .
$$

Thus we complete the proof.

The next lemma improves the convergence of $\nabla \psi^{j_{k}}$ by a nonlinear test function method in [5].

Lemma 2.3. The above weak convergence can be improved to the strong convergence

$$
\nabla \psi^{j_{k}} \rightarrow \nabla \psi \quad \text { in } \quad L_{l o c}^{2}(\Omega)
$$

Proof. Without loss of generality, let us consider the problem on $\Omega_{1}$. We choose a compact set $K$ in $\Omega_{1}$. When we consider the matrix of second order coefficients of (2.6), we find the smallest eigenvalue of the matrix is $\min \left\{\psi^{j_{k}}+g-x, 1\right\}$ which is possibly zero at only $\{x=0\} \subset \partial \Omega$. For simplicity of notations, let $\psi^{j_{k}}$ be $\psi^{j}$.

We choose a non-negative function $\rho \in C_{c}^{\infty}(\Omega)$ such that $\rho=1$ in a compact set $K$ and $\operatorname{supp} \rho \subset \subset \Omega_{1}$. Let $f \in L^{\infty}(\Omega) \cap W_{\text {loc }}^{1,2}(\Omega)$. Multiply (2.6) by $\rho f$ and integrate over $\Omega_{1}$ to get

$$
\begin{gathered}
\int_{\Omega_{1}} \rho f\left\{\left(\psi^{j}+g-x\right) g_{x x}-\left(\psi_{x}^{j}+g_{x}-1\right) \psi_{x}^{j}\right. \\
\left.+g_{y y}+\left(\psi_{x}^{j}+g_{x}\right)\left(\left(\psi_{x}^{j}+g_{x}\right)-\frac{1}{2}\right)\right\} \\
=\int_{\Omega_{1}}\left\{f \rho_{x}\left(\psi^{j}+g-x\right) \psi_{x}^{j}+\rho f_{x}\left(\psi^{j}+g-x\right) \psi_{x}^{j}+\rho_{y} f \psi_{y}^{j}+\rho f_{y} \psi_{y}^{j}\right\} .
\end{gathered}
$$


Let us take $f=\sinh \left[\theta\left(\psi^{j}-\psi\right)\right]$ where $\theta>0$ is to be determined. Then we have

$$
\begin{aligned}
& \int \theta \rho \cosh \left(\theta\left(\psi^{j}-\psi\right)\right)\left(\left(\psi_{x}^{j}-\psi_{x}\right)\left(\psi^{j}+g-x\right) \psi_{x}^{j}+\psi_{y}^{j}\left(\psi_{y}^{j}-\psi_{y}\right)\right) \\
&= \int \rho \sinh \left(\theta\left(\psi^{j}-\psi\right)\right)\left(\left(\psi^{j}+g-x\right) g_{x x}-\left(\psi_{x}^{j}+g_{x}-1\right) \psi_{x}^{j}+g_{y y}\right. \\
&\left.+\left(\psi_{x}^{j}+g_{x}\right)^{2}-\frac{1}{2}\left(\psi_{x}^{j}+g_{x}\right)\right) \\
&-\int \sinh \left(\theta\left(\psi^{j}-\psi\right)\right)\left(\rho_{x}\left(\psi^{j}+g-x\right) \psi_{x}^{j}+\rho_{y} \psi_{y}^{j}\right) .
\end{aligned}
$$

Furthermore, this becomes

$$
\begin{aligned}
& \int \theta \rho \cosh \left(\theta\left(\psi^{j}-\psi\right)\right)\left(\left(\psi_{x}^{j}-\psi_{x}\right)^{2}\left(\psi^{j}+g-x\right)+\left(\psi_{y}^{j}-\psi_{y}\right)^{2}\right) \\
= & \int \rho \sinh \left(\theta\left(\psi^{j}-\psi\right)\right)\left(\left(\psi^{j}+g-x\right) g_{x x}-\left(\psi_{x}^{j}+g_{x}-1\right) \psi_{x}^{j}+g_{y y}\right. \\
\left.+\left(\psi_{x}^{j}+g_{x}\right)^{2}-\frac{1}{2}\left(\psi_{x}^{j}+g_{x}\right)\right) & \\
& -\int \theta \rho \cosh \left(\theta\left(\psi^{j}-\psi\right)\right)\left(\left(\psi_{x}^{j}-\psi_{x}\right)\left(\psi^{j}+g-x\right) \psi_{x}+\psi_{y}\left(\psi_{y}^{j}-\psi_{y}\right)\right) \\
& -\int \sinh \left(\theta\left(\psi^{j}-\psi\right)\right)\left(\rho_{x}\left(\psi^{j}+g-x\right) \psi_{x}^{j}+\rho_{y} \psi_{y}^{j}\right) .
\end{aligned}
$$

Note that $\psi_{x}^{j} \rightarrow \psi_{x}$ and $\psi_{y}^{j} \rightarrow \psi_{y}$ in $L^{2}$ and $\cosh \left(\theta\left(\psi^{j}-\psi\right)\right)\left(\psi^{j}+g-x\right)$ is bounded and converges a.e. as $j \rightarrow \infty$ and $\psi^{j}$ converges everywhere since $\psi^{j} \rightarrow \psi$ in $L_{l o c}^{2}(\Omega)$. Then $\cosh \left(\theta\left(\psi^{j}-\psi\right)\right)\left(\psi^{j}+g-x\right) \psi_{x}$ and $\cosh \left(\theta\left(\psi^{j}-\psi\right)\right) \psi_{y}$ converge strongly in $L^{2}$. Then the third integral of the above equation converges to zero since it is the sum of pairings of weak and strong convergent sequences. Since $\sinh \left(\theta\left(\psi^{j}-\psi\right)\right) \rightarrow 0$ as $j \rightarrow \infty$ strongly and by similar reasons, the fourth integral converges to zero. By the ellipticity condition with the smallest eigenvalue $0 \leq \lambda_{0}:=\min \left\{\psi^{j}+g-x, 1\right\}$ on $\Omega_{1}$, the above integral equation involving four integrals becomes

$$
\begin{aligned}
& \int \theta \lambda_{0} \rho \cosh \left(\theta\left(\psi^{j}-\psi\right)\right)\left|\nabla\left(\psi^{j}-\psi\right)\right|^{2} \\
\leq & o(1)+C \int \rho\left|\sinh \left(\theta\left(\psi^{j}-\psi\right)\right)\right|\left(1+\left|\nabla \psi^{j}\right|^{2}\right),
\end{aligned}
$$

where $C$ is a constant independent of $j$. Since $\left|\nabla \psi^{j}\right|^{2}$ can be replaced by $2|\nabla \psi|^{2}+2\left|\nabla\left(\psi^{j}-\psi\right)\right|^{2}$

$$
\begin{aligned}
& \int_{\Omega_{1}} \theta \lambda_{0} \rho \cosh \left(\theta\left(\psi^{j}-\psi\right)\right)\left|\nabla\left(\psi^{j}-\psi\right)\right|^{2} \\
\leq & o(1)+C \int_{\Omega_{1}} \rho\left|\sinh \left(\theta\left(\psi^{j}-\psi\right)\right)\right|+2 C \int_{\Omega_{1}} \rho\left|\sinh \left(\theta\left(\psi^{j}-\psi\right)\right)\right||\nabla \psi|^{2}
\end{aligned}
$$




$$
+2 C \int_{\Omega_{1}} \rho\left|\sinh \left(\theta\left(\psi^{j}-\psi\right)\right)\right|\left|\nabla\left(\psi^{j}-\psi\right)\right|^{2} .
$$

Similarly to the above method, we have

$$
\begin{aligned}
& \int \theta \lambda_{0} \rho \cosh \left(\theta\left(\psi^{j}-\psi\right)\right)\left|\nabla\left(\psi^{j}-\psi\right)\right|^{2} \\
\leq & o(1)+C \int \rho \cosh \left(\theta\left(\psi^{j}-\psi\right)\right)\left|\nabla\left(\psi^{j}-\psi\right)\right|^{2}
\end{aligned}
$$

since $|\sinh (\tau)| \leq \cosh (\tau)$ for all $\tau$. Since $\rho=1$ on $K$ and $\cosh (\tau) \geq 1$ for all $\tau$, the above inequality becomes

$$
(\theta \lambda-C) \int_{K}\left|\nabla\left(\psi^{j}-\psi\right)\right|^{2} \leq o(1),
$$

where $\lambda$ is the least one between $\min _{K} \delta \phi$ and 1 from (2.4). Taking large enough $\theta>0$, we have the strong convergence of $\nabla \psi^{j}$ to $\nabla \psi$ in $L_{l o c}^{2}(\Omega)$.

Therefore we have a subsequence $\left\{\psi^{j_{k}}\right\}$ converging to a function $\psi \in H_{l o c}^{1}(\Omega)$ with

$$
\psi^{j_{k}} \rightarrow \psi \quad \text { in } \quad L_{l o c}^{2}(\Omega), \quad \text { and } \quad \nabla \psi^{j_{k}} \rightarrow \nabla \psi \quad \text { in } \quad L_{l o c}^{2}(\Omega) .
$$

Then $\psi$ satisfies (2.6) in the sense of distribution.

Now we will show that the solution $\psi$ satisfies the boundary condition using a function $\chi \in C_{c}^{\infty}\left(\mathbb{R}^{2}\right)$ with $\chi=1$ in $\bar{B}(p, r / 2)$ and $\chi=0$ outside $B(p, r)$ for a point $p \in \partial \Omega$. Let us call $\chi$ a localizer for a point on the boundary.

Lemma 2.4. The solution $\psi$ satisfies the boundary condition in the sense

$$
\chi \psi^{3 / 2} \in H_{0}^{1}(\Omega),
$$

where $\chi=\chi(\cdot ; p, r)$ is a localizer for a point $p \in \partial \Omega$.

Proof. Define $S=\Omega \cap B(p, r)$ and $\psi^{j_{k}}:=\psi$. In this proof $C$ denotes a constant depending on $S$.

It is clear that $\int_{S}\left|(\nabla \chi) \psi^{3 / 2}\right|^{2}<C$ by the property of $\chi$. Similarly to the method in the proof of Lemma 2.2, we can show that $\int_{S} \chi^{2} \psi|\nabla \psi|^{2}<C$. As in (2.7), we use the zero boundary conditions of $\psi$ and $\chi$ to get

$$
\begin{aligned}
\int_{S} \chi^{2}\left(\psi_{x}^{2}(\psi+g-x)+\psi_{y}^{2}\right) d x d y & \int_{S}\left\{\chi^{2} \psi(\psi+g-x) g_{x x}-2 \chi \chi_{x} \psi(\psi+g-x) \psi_{x}+\chi^{2} \psi \psi_{x} g_{x}\right. \\
& \left.\quad+\frac{1}{2} \chi^{2} \psi \psi_{x}+\chi^{2} g_{y y} \psi-2 \chi \chi_{y} \psi \psi_{y}+\chi^{2} g_{x}^{2} \psi-\frac{1}{2} \chi^{2} \psi g_{x}\right\} d x d y
\end{aligned}
$$

If we use the Cauchy inequality with $\varepsilon$, the above equation becomes

$$
\int_{S}\left((\psi+g-x) \chi^{2} \psi_{x}^{2}+\chi^{2} \psi_{y}^{2}\right) \leq C+C \varepsilon \int_{S} \chi^{2} \psi \psi_{x}^{2}
$$


We note that $\psi:=\psi^{j_{k}}$ is uniformly bounded in $S$ so there exists some number $L_{0}>0$ such that $0 \leq \psi / L_{0}<1$ in the domain. Then the fact that $\psi:=u-g \leq$ $u-x$ in $S$ for sufficiently small $r>0$ yields

$$
\int_{S}\left(\chi^{2} \psi \psi_{x}^{2}+\chi^{2} \psi \psi_{y}^{2}\right) \leq C+C \varepsilon \int_{S} \chi^{2} \psi \psi_{x}^{2}
$$

So if we take $\varepsilon>0$ small enough such that $C \varepsilon<<1$, then $\int_{S} \chi^{2} \psi|\nabla \psi|^{2} \leq C$. Thus $\int_{S}\left|\nabla\left(\chi\left(\psi^{j_{k}}\right)^{3 / 2}\right)\right|^{2} d x d y<C$. And for all sufficiently large $j_{k}, \chi \psi^{j_{k}}=0$ on $\partial S$. Then for the limit function $\psi$ we have

$$
\int_{S}\left|\nabla\left(\chi \psi^{3 / 2}\right)\right|^{2} d x d y<C,\left.\quad \chi \psi^{3 / 2}\right|_{\partial S}=0
$$

in trace sense. Hence the solution $\psi$ takes its boundary value in the sense of $\chi \psi^{3 / 2} \in H_{0}^{1}(\Omega)$ for a localizer $\chi$.

Therefore, using all of these lemmas we lead to Theorem 1.1 which says that there exists a weak solution $u \in H_{l o c}^{1}(\Omega)$ of (1.7) and the solution takes its boundary values in the sense $\chi(u-g)^{3 / 2} \in H_{0}^{1}(\Omega)$.

\section{References}

[1] M. Brio and J. Hunter, Mach reflection for the two-dimensional Burgers equation, Physica D 60 (1992), no. 1-4, 194-207.

[2] S. Canić, B. L. Keyfitz, and E. Kim, A free boundary problem for a quasi-linear degenerate elliptic equation: regular reflection of weak shocks, Comm. Pure Appl. Math. 55 (2002), no. 1, 71-92.

[3] S. Canić, B. L. Keyfitz, and G. Lieberman, A proof of existence of perturbed steady transonic shocks via a free boundary problem, Comm. Pure Appl. Math. 53 (2000), no. 4, 484-511.

[4] S. Canić and E. Kim, A class of quasilinear degenerate elliptic problems, J. Differential Equations 189 (2003), no. 1 , 71-98.

[5] L. Evans, Weak Convergence Methods for Nonlinear Partial Differential Equations, CBMS Regional Conference Series in Mathematics, 74. Published for the Conference Board of the Mathematical Sciences, Washington, DC; by the American Mathematical Society, Providence, RI, 1990.

[6] C. S. Morawetz, Potential theory for regular and Mach reflection of a shock at a wedge, Comm. Pure Appl. Math. 47 (1994), no. 5, 593-624.

[7] K. Song, A pressure-gradient system on non-smooth domains, Comm. Partial Differential Equations 28 (2003), no. 1-2, 199-221.

[8] E. Tabak and R. Rosales, Focusing of weak shock waves and the von Neumann paradox of oblique shock reflection, Phys. Fluids A 60 (1994), no. 5, 1874-1892.

[9] Y. Zheng, Existence of solutions to the transonic pressure-gradient equations of the compressible Euler equations in elliptic regions, Comm. Partial Differential Equations 22 (1997), no. 11-12, 1849-1868.

[10] _ Two-dimensional Riemann problems for systems of conservation laws, Birkhäuser, 2001.

Department of Mathematics

KYUNG HeE UNIVERSiTy

SEOUl 130-701, KOREA

E-mail address: kyusong@khu.ac.kr 